\title{
DISCLAIMER
}

This report was prepared as an account of work sponsored by an agency of the United States Government. Neither the United States Government nor any agency thereof, nor any of their employees, makes any warranty, express or implied, or assumes any legal liabiiity or responsibility for the accuracy, completeness, or usefulness of any information, apparatus, product, or process disclosed, or represents that its use would not infringe privately owned rights. Reference berein to any specific commercial product, process, or service by trade aame, tradernark, manufacturer, or otherwise does not necessarily constitute or imply its endorsement, recommendation, or favoring by the United Siates Goverrment or any agency thereof. The views and opinions of authors expressed herein do not necessarily state or reflect those of the United States Covernment or any agency thereof.

POSSIBILITIES FOR X-RAY HCLOGRAPHY USJNG SYNCHROTRON RADIATION

M.R. Howe 11 s

BHL -33994

DESE 010390

November 1983

Research Supported by the OFFICE OF BASIC ENERGY SCIENCES

U.S. DEPARTMENT OF ENERGY WASHINGTON, D.C.

\footnotetext{
NATIONAL SYNCHROTRON LIGHT SOURCE BROOKHAVEN NATIONAL LABORATORY Associated Universities, Inc.

Under contract No. DE-ACO2-76CH00016 with the United States Depirtment of Energy
} 
Possibilities for X-Ray Holography Using Synchrotron Radiation

\author{
M. R. Howe $11 \mathrm{~s}$ * \\ Brookhaven National Laboratory \\ Upton, New York 11973
}

\title{
1. Introduction
}

Since the theoretical $(1)$ and experimental $(2,3)$ demonstrations of the effectiveness of soft $x$-rays in imaging biological material there has been considerable study $(4,5)$ given to the prospects for further development of the presently existing techniques. This has been motivated to a large extent by advances in source technology, particularly the use of undulators on electron storage rings and recent improveceiss in short wavelength lasers. The present author has carried out theoretical evaluations of the possibilities of holographic imaging and has also recorded a number of holograms using the 015 soft $x$-ray beamline at the National Synchrotron Light Source (NSLS) $750 \mathrm{MeV}$ storage ring at Brookhaven. Some of these have been successfully reconstructed using He:Cd laser light.

In this paper we first revies the physical processes which generate information containing wavefronts when soft $x$-rays interact with matter. We then briefly describe the holographic method which has been highly developed using visible light lasers and identify holographic geometries which are promising for $x$-ray applications. We discuss some of the practical and theoretical limitations involved in making holographic images and then give the results of our own experiments.

\section{Soft $\mathrm{X}-\mathrm{Ray}$ Interactions with Matter}

The situation of interest is represented in Fig. 1. Soft $x$-rays are incident fram the left as a plane wave of unit amplitude. They interact with the sample which is represented by a refractive index distribution $n(\underline{I})=1-\delta(\underline{I})-i \beta(\underline{r})$ and are received by the detector where we wish to make a holographic recording. We know that in free space the amplitude $U(\underline{I})$ of the wave must satisfy the Helmholtz equation

$$
\nabla^{2} \psi+k_{0}^{2} \psi=0
$$

where $k_{0}$ is the free space propagation constant. In the sample $k_{0}$ becomes $\mathrm{nk}_{\mathrm{o}}$ and (1) becomes

\footnotetext{
*This work was supported by the U.S. Department of Energy unaer concract number DE-ACO2-76-CHOOO16.
} 


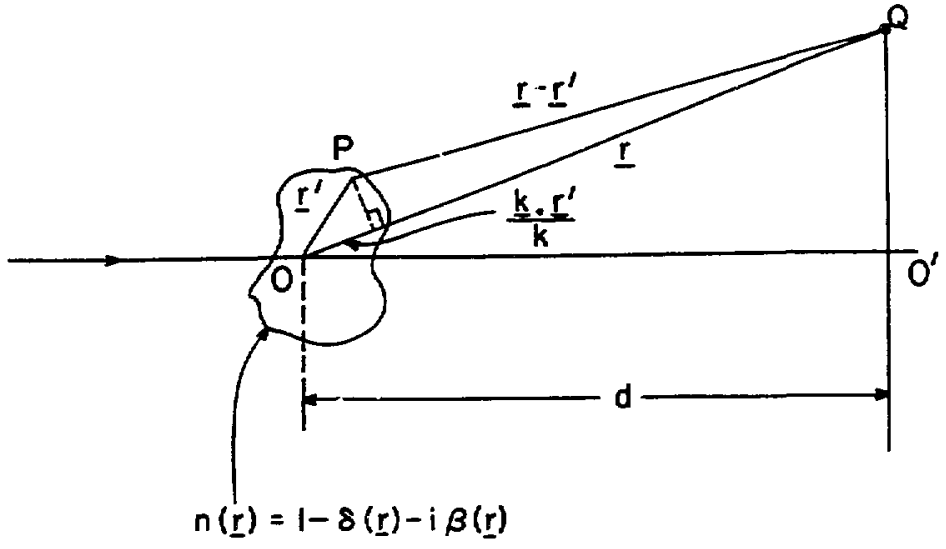

Fig. 1. Notation for discussion of soft $x$-ray interactions with a refractive index distribution $n(\underline{r})$

$$
\nabla^{2} \psi+k_{0}^{2} \psi=\left(1-n^{2}\right) k_{0}^{2} \psi=0 \psi
$$

This is a familiar equation and it is a standard result (6) that the solpution is

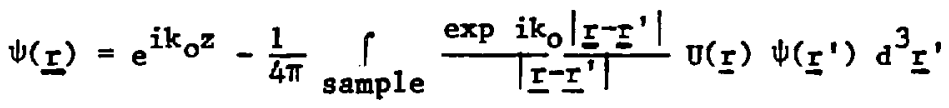

For particle scattering by a potential $V(r), U(r)$ would be $\left(8 \pi^{2} \mathrm{~m} / \mathrm{h}^{2}\right) \nabla(\underline{r})$. For scattering of $x$-rays by a charge distribution of $\rho(\underline{r})$ electrons per unit volume, $U(I)$ is $4 \pi r_{0} \rho(I)$ where $r_{0}$ is the classical electron radius. For the present case $v(r)=\left(1-n^{2}\right) k_{0}{ }^{2}$. In all these cases (3) tells us that at each volume element $\bar{d}^{3} r^{\prime}$ the wave is depleted by the scattering of a secondary wave let of srength, $-U\left(\underline{r}^{\prime}\right) / 4 \pi$ times the value of the incident amplitude at that point. In our case $\mathrm{U}(\underline{r})$ in general is complex so that both absorption and scattering are expected to occur. For conventional $x$-ray diffraction (3) is interpreted by making two idealizations: (i) the Fraunhofer approximation, $w^{2} / \lambda_{d}<<1$ where $w$ is the width of the sample. In other words the detector is assumed to be in the far field of the sample; and ( $i i)$ the Born approximation, the use of the unmodified incoming wave as an approximation to $\psi\left(I^{\prime}\right)$ in (3). It is helpful at this point to define unit vectors $s_{0}$ and $\underline{s}$ in the incoming and outgoing (DQ) directions, respectively. With these assumptions $k_{0}\left|r-r^{\prime}\right|$ in the exponent becomes $k_{0} r-k_{0} s \cdot I^{\prime}$ and using the definition $\underline{R}=\bar{k}_{0}\left(\underline{s}_{0}-\underline{s}\right)$ (3) can be written (dropping the prime)

$$
U(\underline{I})=e^{i k_{0} z}-\frac{e^{i k_{0} I}}{4 \pi \tau} \int_{s a m p l e} U(\underline{I}) e^{i \underline{R} \cdot I} d^{3} \underline{I}
$$

(4) is of the form of an asymptotic solution $e^{i k_{0} z}+f\left(A_{x}, \theta_{y}\right) e^{i k_{0} I / x}$ so we can identify $f\left(A_{x},{ }^{A}\right)$ and see that 


$$
\frac{d \sigma\left(K_{x}, K_{y}\right)}{d \Omega}=\left|f\left(\theta_{x},{ }_{y}\right)\right|^{2}=\frac{1}{16 \pi^{2}}\left|\tilde{u}\left(R_{x,} R_{y}\right)\right|^{2}
$$

where

$$
\mathrm{K}_{\mathrm{x}}=\mathrm{k}_{\mathrm{o}} \sin \theta_{\mathrm{x}}, \mathrm{K}_{\mathrm{y}}=\mathrm{k}_{\mathrm{o}} \sin \theta_{y}, \mathrm{k}_{z}=-\mathrm{k}_{\mathrm{o}}\left(1-\sqrt{1-\sin ^{2} \theta_{\mathrm{x}}-\sin ^{2} \theta_{y}}\right)
$$

and $Q$ (Fig. 1) is the point $\left(r \sin \theta_{x}, r \sin \theta_{y}\right)$. This defines the intensity at $Q(x, y)$ in terms of the original function $n(r)$ within the limitations of the Fraunhofer and Born approximations. We note that the Fraunhofer approximation is not valid in the normal optical regime where the more restrictive Fresne 1 approximation $(\theta$ sma 11$)$ is used (13). To see the relation of our refractive index picture to the atomic picture implied in $\rho(\underline{r})$ we note that according to Henke $(7) \delta$ and $B$ are related to $f_{1}$ and $f_{2}$, the real and imaginery parts of the atomic scattering factor by

$$
\delta=\frac{r_{0} \lambda^{2}}{2 \pi} n_{0} f_{1} \quad, \quad B=\frac{r_{0} \lambda^{2}}{2 \pi} n_{0} f_{2}
$$

where $n_{0}$ is the number of atoms per unit volume. So

$$
U(\underline{I})=k_{0}^{2}\left(1-n^{2}\right) \simeq 2 k_{0}^{2}(\delta+i B)
$$

inserting (7) into (8) gives

$$
U(\underline{r}) \simeq 4 \pi r_{0} n_{0}\left(f_{1}+i f_{2}\right) \equiv 4 \pi r_{0} \rho(\underline{r})
$$

as it should where now $\rho(\underline{r})$ is understood as the effective number of electrons per unit volume.

of course it is not normally possible to obtain $U(\underline{r})$ from $\left|\tilde{v}\left(k_{x}, K_{y}\right)\right|^{2}$ without prior knowledge of $U(\underline{r})$ and such prior knowledge is not expected to be available in microscopy. In order to obtain $U(\underline{r})$ from $\left|\tilde{U}\left(R_{x}, R_{y}\right)\right|^{2}$ we need a method of determining the phase of $W(I)$ at each point $Q$. The holographic approach to this is to beat the waves $U(\underline{I})$ against a standard reference wave. The resulting interference fringes give a full record of the phases of $U(\underline{r})$ at all points on the detector. In addition, if the recording is suitably made, it can be used as a diffracting structure. In this case illumination by the original reference wave gives the diffraction pattern of the diffraction pattern and reconstructs the original optical density distribution of the sample. We will enlarge on this later. The process is similar to the Bragg microscope $(8)$ and to optical transform methods (9) used in crystallography. If the holographic recording is inverted optically then it is not subject to the limitations of the Fraunhofer and Born approximations and would in principle allow a perfect reconstruction if all spatial frequencies $K_{x}, K_{y}$ present in the sample were recorded. However, as we shall see there are some fundamental difficulties 
in providing reference waves over the entire Ewald sphere. Generally speaking the holographic method should be complementary to the alternative of measuring the entire diffraction pattern without the use of reference waves. For holography, high frequency information is lost due to incomplete collection of the diffraction pattern. For the diffraction approach the methods for determining the phases will be the limitation.

\section{Resolution Limitations Due to Beam Geometry}

From (6) we see directly that a feature of width $\delta x$ corresponding roughly to a frequency $2 \pi / \delta x$ leads to a diffracted beam at $e_{x}$ where

$$
\mathrm{k}_{\mathrm{x}}=\frac{2 \pi}{\delta \mathrm{x}}=\frac{2 \pi}{\lambda} \sin \theta_{\mathrm{x}}
$$

We recognize the grating equation $\lambda=\delta x \sin ^{A} x$ and we see clearly the relation between transverse resolution and the diffracted bean angle, i.e. for $\delta x$ to be small, as desired, $P_{x}$ must be large.

We could make a similar argument from (6) for $K_{z}$ and get the 1 imit of the longitudinal resolution. However, we prefer to make an optical argument. It is a standard result of geometrical optics that the wavefront aberration $(\delta w)$ for the edge rays of a pencil of half angle $A$ whose source point is subject to a longitudinal focal shift $\delta z$, is given by

$$
\delta W=\frac{3}{2} A^{2} \delta_{z}(\theta \operatorname{sma} 11)
$$

If we accept that the Rayleigh quarter wave criterion gives the range of wavefront deformations which are not detectable at the image then we can set $\delta W=\frac{1}{4} \lambda$ and get $a \delta_{z}$ which is a measure of the longitudinal resolution

$$
\delta z= \pm \frac{\lambda}{2 e^{2}}
$$

To get an idga of scale suppgse $\theta=0.1$ radians and $\lambda=30 \AA$. We find $\delta_{x}, \delta_{y} \simeq 300$ A but $\delta_{z}=1500$ A. Using (6) gives essentially the same result: $\delta z=3000$ A. So we see without even considering how to do holography that its potential for gathering three dimensional information is strictly limited by the collection angle and that through (9) and (11) or (6) the transverse and longitudinal resolution are related.

\section{The Holographic Method (10-13)}

A generalized view of a holographic layout is shown in Fig. 2. The subject is illuminated by waves from a source. At the same time a reference object is illuminated by the same source in a manner which maintains coherence over the whole wavefront reaching both objects. The waves are scattered by the objects so that a wavefront from the subject arrives at the hologram recording plane, leading to an amplitude distribution $a_{s}(x, y)$ in that plane. 


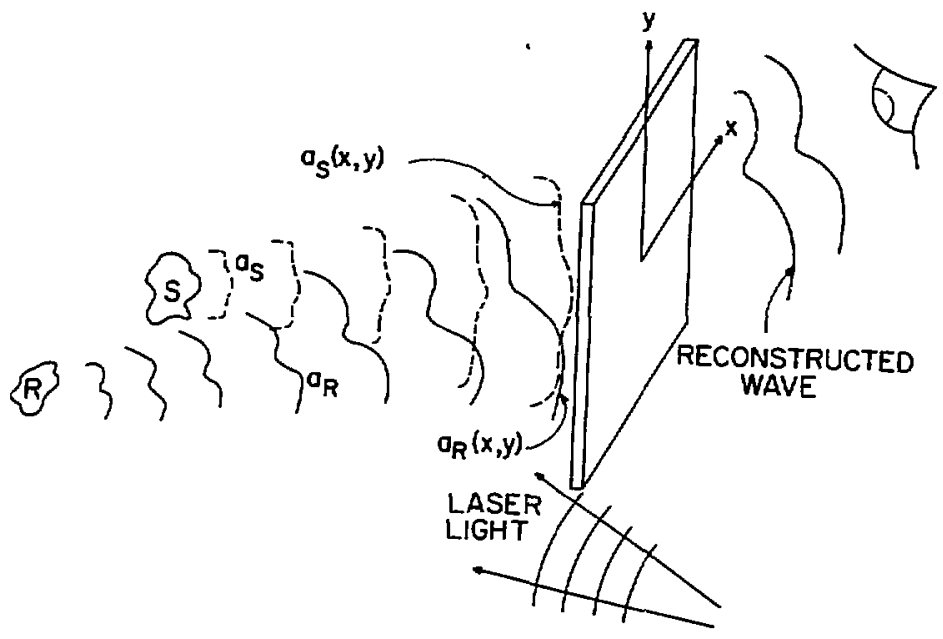

Fig. 2. Combination of a subject and reference wavefront to give an interference pattern. The amplitude functions $a_{\mathbf{s}}(a x, y)$ and $a_{R}(x, y)$ refer to amplitude distributions at the hologram plane

Similarly another wavefront from the reference object gives an amplitude $a_{R}(x, y)$. The amplitudes add and give rise to an intensity distribution $I=\left(a_{s}+a_{R}\right)\left(a_{s}{ }^{*}+a_{R}{ }^{*}\right)$. This is recorded, for example, photographically and developed to give an amplitude transmittance $(t)$ proportional to the exposure, i.e.

$$
t \alpha I=a_{s} a_{s}^{*}+a_{R} a_{R}^{*} * a_{s} a_{R}^{*}+a_{s}{ }^{*} a_{R}
$$

Now suppose we illuminate the hologram with the original reference wave. The amplitude just past the hologram $w$ is given by

$$
\begin{aligned}
& \begin{aligned}
w=a_{R} t \alpha & a_{R}\left|a_{s}\right|^{2} \\
+ & a_{R}\left|a_{R}\right|^{2}
\end{aligned} \quad \text { intermodulation term } \quad\left\{\begin{array}{l}
\text { Zero } \\
\text { order } \\
\text { terms }
\end{array}\right. \\
& +a_{s}\left|a_{R}\right|^{2} \text { VIRTUAL IMAGE TERM } \\
& +a_{R^{a}} R_{s}^{*} \text {. Confusing wave }
\end{aligned}
$$

The third term is an aberration free reconstruction of the wavefront which previously was emitted directly by the subject. The fourth term, can, under suitable conditions, lead to formation of a real image of the subject but from the point of view of observing the virtual image it is a nuisance.

We may note the following general points: (i) (12) is symetrical in as and $a_{R}$. So illumination of the hologram by either one reconstructs the other. (ii) Although, according to the above, any wave can be the reference wave, it is much more convenient to use plane or spherical waves. These can be recreated anytime without needing to re-use the reference object. (iii) Reconstruction need not be with the original reference wave. If the reference source was a point (including the point at infinity) then any 
point source will reconstruct an. image of the subject. However, if the wavelength or the source to hologram distance or the off axis angle is changed then, in general, the magnification will no longer be unity and aberrations are introduced. Similarly, if the hologram is scaled up or down in size the same effects occur. This is important for us because we wish to make a recording with $x$-rays and do the reconstruction in the visible in order to get magnification. There are some special cases where aberrations are small or zero and we return to these later.

\section{Hologram Recording Geometries}

If we make the simplification of considering a point subject and point reference source then we can draw a usefil representaton of the interference fringes that would result if both sources emit coherently in all directions. This is shown in Fig. 3. We see that there is a rich variety of geometries with which visible light holograms can be recorded. Only three of these are of interest for $x$-ray use: the Gabor in-line hologram $(14)$, the LeithUpatneiks off-axis hologram $(15)$ and the Fourier Transform hologram $(16)$. We consider them in turn.

The Gabor in-line hologram uses the light that happens to be transmitted through the sample as the reference beam. For the Gabor hologram, each point of the subject contributes an amplitude distribution in the hologram which is a complete circular Fresnel zone plate pattern centered near the axis. The advantage is that no optics are needed except a pinhole. The most obvious disadvantages are that the sample must be chosen to transmit suitably and there is no good solution to the problem of separating the wanted wavefront from the confusing wave. This problem can, however, be much alleviated by placing the recording surface in the far field of the sample $(17)$. The far field or Fraunhofer in-line geometry was

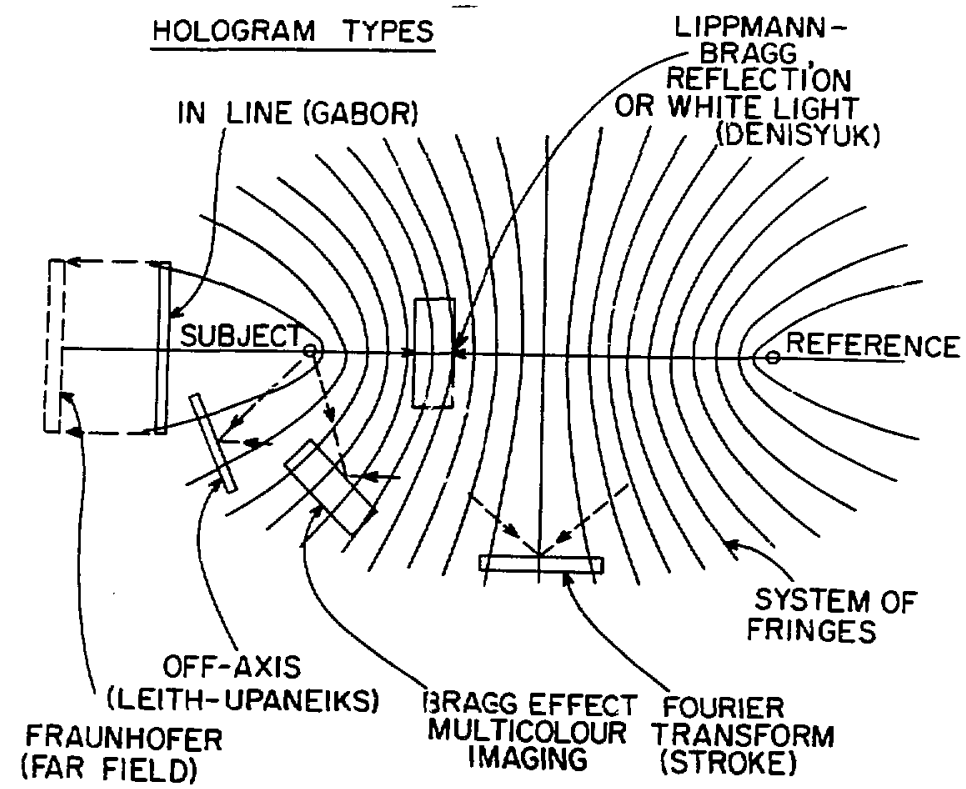

Fig. 3. Idealized fringe distribution due to two point sources with indication of the regions used for various types of visible light holograms 
the one used by Aoki and Rikuta $(30)$ in recording what are probably the best $x$-ray holograms made with a microfocus tube.

The Leith-Upatneiks hologram is similar to the Gabor but an off-axis reference beam is used. In this case each point of the subject contributes an off-axis zone plate. The center may be off the recording area and so higher numbered, more closely spaced zone plate rings are utilized. The advantage is that separation of the virtual image wave and the confusing wave can be achieved. The disadvantage is that a higher resolution detector and a source with greater coherence length are needed. Some optics are also required.

The Fourier Transform hologram is a radically different approach. Here the reference is typically a point source and must be set at a distance from the hologram equal to that of the subject. The subject-reference distance is chosen about equal to the subject width which in microscopy would be sma11. The interfering beams thus have only a small angle between them so rather coersly spaced Youngs fringes are recorded on the detector. Consequently the resolution is not limited by the detector resolution but rather by the actual size of the nominal point source used as a reference or by the considerations of Section 3. Detector resoiution plays a role in limiting the field of view.

In Fig. 4 we indicate ways in which the three recording geometries just discussed might be used to make $x$-ray holograms.

(a)

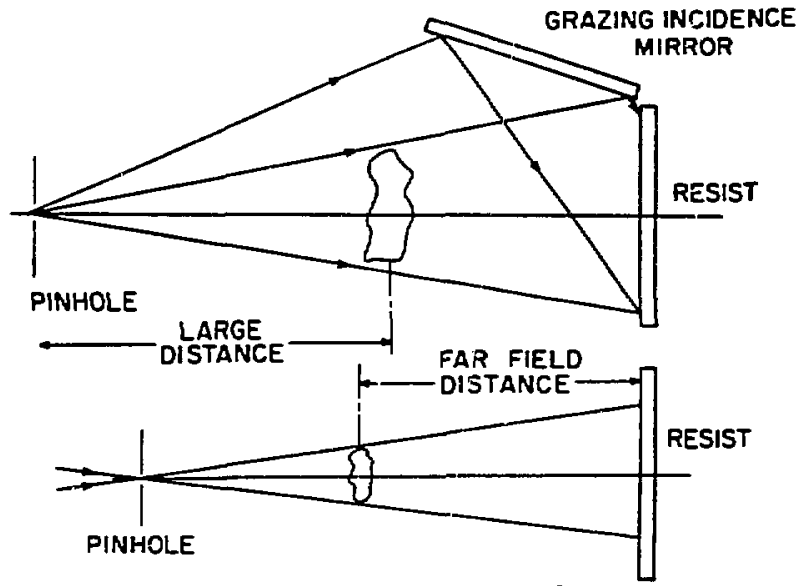

(b)

(c)

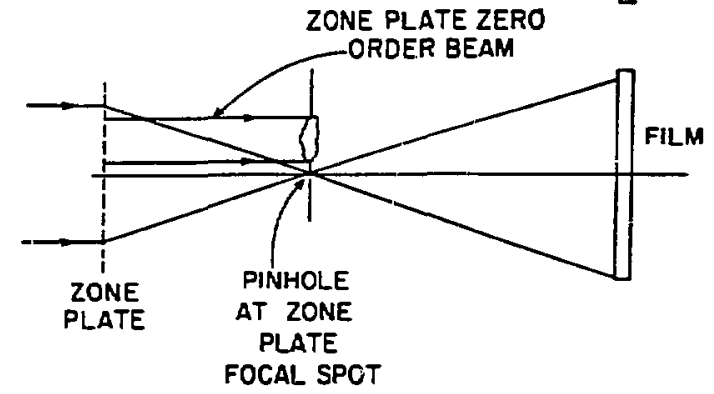

Fig. 4. Possible geometries for x-ray holography utilizing (a) LeithUpatneiks geometry, (b) Gabor geometry, (c) Fourier Transform Geometry 


\section{The Paraxial Oetics of Point Source Holograms}

Consider a subject point $\left(x_{1}, y_{1}, z_{1}\right)$ and a reference poirt $\left(x_{R}, y_{R}, z_{R}\right)$ in a co-ordinate frame defined so that the recording surface is the plane $z=0$. Suppose a hologram is formed with light of wavelength $\lambda_{1}$ and reconstructed with light of wavelength $\lambda_{2}\left(\lambda_{2} / \lambda_{1}=\mu\right)$. Let the reconstruction source be at $\left(x_{c}, y_{c}, z_{c}\right)$ and suppose the real and virtual images are at $\left(x_{3 R}, y_{3 R}, z_{3 R}\right)$ and $\left(x_{3 V}, Y_{3 V}, z_{3 V}\right)$ respectively. Furthermore, let the hologram be scaled up by a factor m before reconatruction. It is now a standard result $(10,11)$ that the positions of the images are given by

$$
\frac{1}{z_{3 V, R}}=\frac{1}{z_{c}} \pm \frac{\mu}{m^{2} z_{1}} \mp \frac{\mu}{m^{2} z_{R}}
$$

The upper signs refer to $z 3 v$, the lower to $z_{3 R}$. Also the lateral magnification $\mathrm{M}_{\text {LAT }}$ is given by:

$$
M_{\text {LAT }, v, R}=\frac{m}{1 \pm \frac{m^{2} z_{1}}{\mu z_{c}}-\frac{z_{1}}{z_{R}}}=\frac{\mu}{m} \frac{z_{3 V, R}}{z_{1}}
$$

where a similar sign convention applies.

Since we are interested in microscopy (15) is an important formula. It was by special choices of the parameters in (15) that Gabor originally intended to achieve high magnification ig his 'projection method.' We are also seeking to use a high value for $\mu$ and achieve magnification. However, it is clear by inspection of (15) that high $\mu$ alone does not guarantee a high value of $M_{L A T}$. There are two ways to achieve this: with $z_{1} \neq z_{R}$ (Gabor and Leith-Upatneiks case) and supposing $m=1$ then $M_{L A T, R}+\infty$ if

$$
\frac{z_{1}}{\mu_{z_{c}}}+1+\frac{z_{1}}{z_{R}}
$$

This does not really depend on high $\mu$ since $z_{c}$ can, in principle, always be chosen to satisfy (16). $z_{1}=z_{R}$ (Fourier Transform case). Then setting $z_{c}=z_{1}=z_{R}$ leads to $M_{L A T, V}=\mu / m$, and this is a promising approach for $x$-ray work. The coarse fringes obtaining in this cse make recording with photographic film and reconstructing with visible light perfectly practical. We note that the conditions for this are

$$
\text { Fringe spacing }=\frac{\lambda_{1}}{\Phi}>\lambda_{2}
$$

where $\phi$ is the angle between the beans. For example if $\lambda_{1}=30 \AA$ and $\lambda_{2}=$ $6328 \mathrm{~A}$ then we find $\phi>5 \mathrm{mR}$, which is quite reasonable. The leading problems remaining in this approach are the beam forming optics for the reference source and the dynamic range of the detector. By way of summary we show in Fig. 5 the three important hologram types and the corresponding 
fringe frequencies in the hologram. It is obvious that from the viewpoint of detector resolution the Leith-Upatneiks case is the most demanding. In fact, if separation of the two images is required the fringe frequency will be four times the highest sample frequency. The Fourier Transform approach has a great advantage in this respect.

\section{Source Coherence Requirements}

The original 'protection method' $\{14\}$ of Gabor was defeated by lack of a suitable source. Even the visible light work which was successfully carried out in the forties and fifties did not make a great impact until the invention of the laser. Sinilarly the development of x-ray holography has been limited up to the present time by lack of a suitable source. However, we believe this is changing. So far we do not have any sources which generate soft $x$-rays in a way which naturally makes all of the radiation coherent in the manner of a laser. Even on this matter, however, there are possibilities for progress (18). For the time being we must still use methods which isolate the coherent part of the output of an incoherent source but much better sources are becoming available.

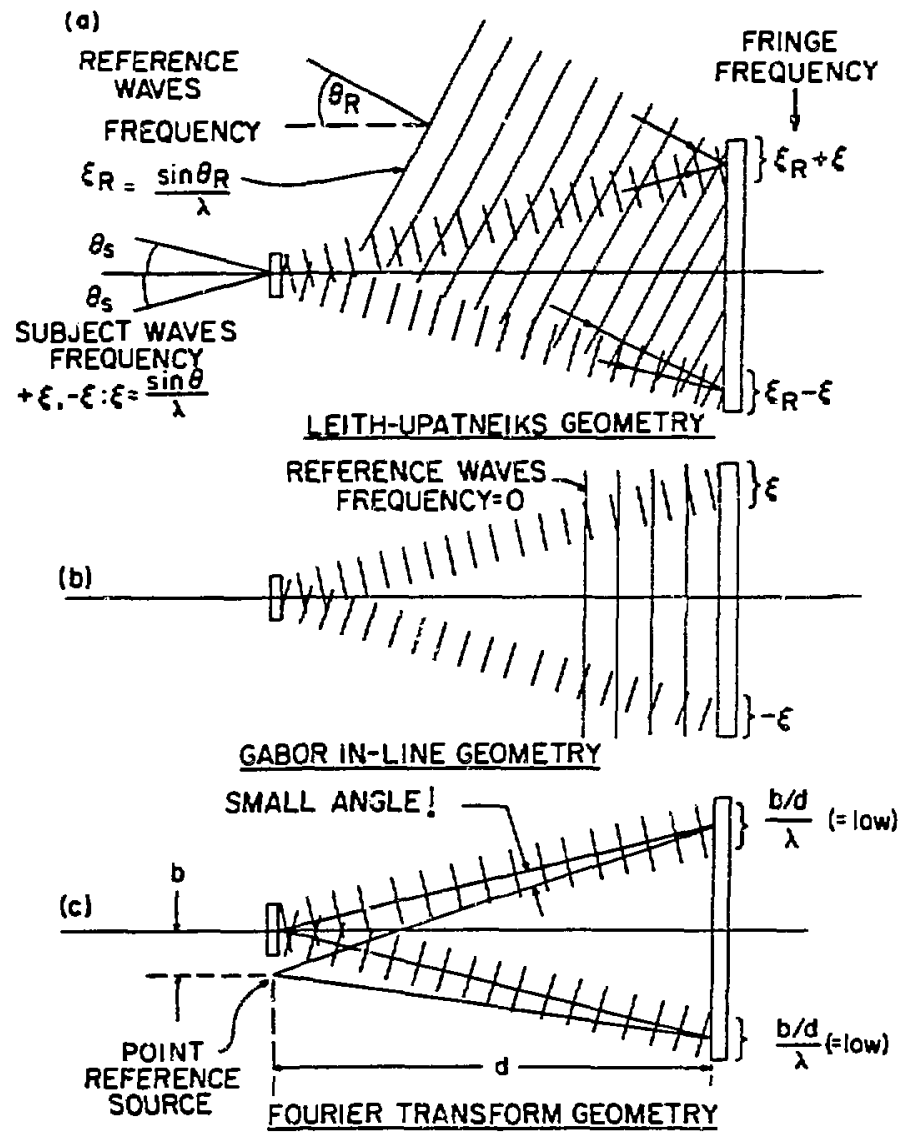

Fig. 5. Fringe frequencies produced by the three main holographic geometries: (a) Leith-Upatneiks, (b) Gabor, (c) Fourier Trans form 
According to the Van Cittert-Zernike Theorem (19) the output of any source is spatially coherent within a region defined by the first minimum of the Fraunhofer Diffraction Pattern of the spatial distribution of the source. For example, if the source is circular and of radius $x$ then spatial coherence exists with in the Airy Cone (of half angle $x^{\prime}$ ). This sets an upper limit on the emittance $\varepsilon$ of a coherent beam given by $(20)$

$$
\varepsilon_{\max }^{c}=x x^{\prime}=0.61 \lambda
$$

This is a very small emittance and (18) sets a strong limitation on the fraction of the output of most sources which is useable for coherent experiments. For example at $\lambda=30 \AA, c_{\max }^{c}=18.0 \AA$.Radians $\equiv 1.8 \mathrm{mR} . \mu \mathrm{m}$. We see that for a good storage ring with $x \simeq 100$ jm we can only utilize $2 x^{\prime}$ $\simeq 2 \varepsilon_{\max }^{c} / 100=0.036 \mathrm{mR}$ ! Equation (18) applies to both dimensions of the beam so the figure of merit of the source for coherent experiments is the flux per unit bandwidth per unit phase space volume. In other words the brightness.

This takes care of spatial or transverse coherence. We now turn to temporal or longitudinal coherence. The requirement here is that the coherence length $\ell_{c}=\lambda^{2} / \Delta \lambda$ should be sufficiently long to coherent $1 y$ illuminate the entire depth of the specimen and should also be greater than the largest path difference between any pair of interfering beams (e.g., $P Q$ in Fig. 6). In view of the penetrating power of soft $x$-rays in biological material we would like to have a source with $\ell_{c}$ at least equal to $1 \mu \mathrm{m}$ and preferrabiy in the region of $3-5 \mathrm{\mu m}$. At $30 \AA \ell_{c}=1 \mathrm{\mu m}$ implies $\lambda / \Delta \lambda \simeq 300$ which is readily achieved using existing soft $x$-ray monochromator technologg. For $l_{c}>3 \mu \mathrm{m}$ then $\lambda / \Delta \lambda>1000$ which can be achieved on $1 \mathrm{~g}$ with considerable effort at the present time.

We see that in principle there is no difficulty in achieving the needed coherence for microscopy of biological specimens. However, in practice, it is lack of flux which presently sets the limit to soft $x$-ray holographic imaging and to this question we now turn.

\section{Flux Requirements}

In order to understand the flux needs of a Gabor hologram, consider the arrangement shown in Fig. 6. A spatially coherent beam of flux $I_{c}$

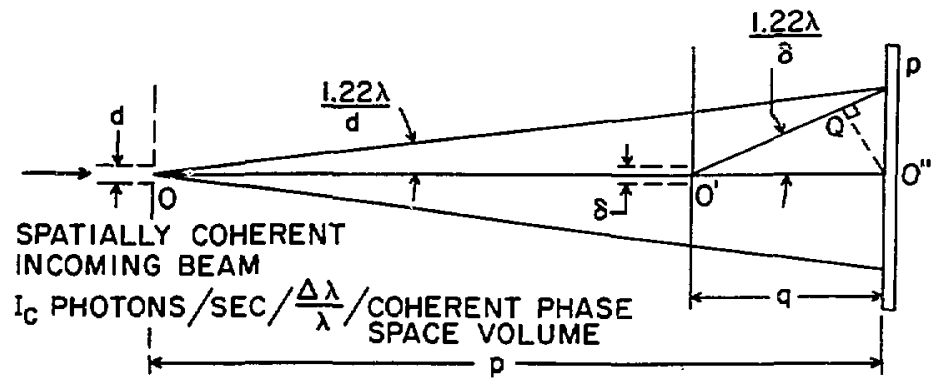

Fig. 6. Layout of imaginary experiment to compute the flux needs of a Gabor hologram 
photons $/ \mathrm{sec} / \Delta \lambda / \lambda$ is focussed on to a pinhole of diameter d at 0 distance $p$ from the detector. By definition the beam will just fill the Airy Cone of the pinhole. This beam is used to illuminate a subject consisting of only one element: a circular hole of diameter $\delta$. The latter is surrounded by an absorbing screen which transmits just enough to provide a reference beam. The Airy Cone of $\delta$ illuminates an area of the detector of radius $r_{n}=1.22$ $\lambda q / \delta$ where $q$ is the working distance. The result is the creation of a Fresne 1 Zone Plate pattern of radius $I_{n}$. According to (9) we must utilize the full Airy Cone of $\delta$ if we are to reconstruct it without loss of resolution. We therefore choose a large value of $p$ which satisfies

$$
\frac{\mathrm{p}}{\mathrm{d}}=\frac{\mathrm{q}}{\delta}
$$

so that the coherent beam exactly fills the circle of radius $r_{n}$. The focal length $f$ of the zone plate is actually given by

$$
\frac{1}{f}=\frac{1}{q}+\frac{1}{p}
$$

but we use the approximation $f \simeq q$.

Now in order for the zone plate to work properly in reconstruction of the subject $\delta$ we need each distinguishable element to be registered with adequate signal to noise ratio. Suppose signal/noige $=5$ is considered adequate. Then each element of detector of area $\Delta r^{2}$ ( $\Delta r$ is the outer ring spacing of the zone plate) should end up registering at least 25 photors from the subject. This will be achieved if

$$
I_{c}\left(\frac{\delta^{2}}{4 r_{n}{ }^{2}}\right)\left(\frac{\Delta r^{2}}{\pi r_{n}{ }^{2}}\right) \cdot 2 Q t \geq 25
$$

where $Q$ is the detective quantum efficiency of the detector, $t$ is the time, and the two arises because only the bright fringes need to receive light. How we know from zone plate theory that $\delta=2.44 \Delta \mathrm{r}$ and $r_{n}=1.22 \lambda_{\mathrm{q}} / \delta$ and for both film and resist $Q=0.2$ is a reasonable estimate so we end up with an estimate of the fluence $I_{c} t$ needed to record the elementary hologram. (Of course if the sample had additional elements these would also contribute zone plate amplitude patterns without needing any more flux.)

$$
I_{c} t \geq R_{1} \frac{\lambda^{4} q^{4}}{\delta^{8}}
$$

where $R_{1}=1.0 \times 10^{4}$. This may also be written

$$
I_{c} t \geq R_{2} n^{4}
$$

where $n$ is the number of zones and $k_{2}=2.0 \times 10^{3}$. 
So far we have regarded $q$ as ar arbitrary parameter but we see from (22) that it has a strong effect on the fluence. The choice of $q$ in a rea 1 hologram recording or a real 1 attempt to manufacture a zone plate would depend on a number of issues which we prefer not to open in the present discussion. Instead we simply plot some sample values of the fluence for interesting values of $\delta$ and $q$ (see Fig. 7). We note that these figures apply to a high contrast subject feature.

Now suppose that we are dealing with Fourier Transform geometry. For this case the sample plane in Fig. 6 will contain two pinholes of diameter $\delta$, separated by a distance $b$ (say). The illuminated circle must now be of diame ter $b$ and (21) becomes

$$
I_{c}\left(\frac{\delta^{2}}{b^{2}}\right)\left(\frac{\lambda^{2} q^{2}}{b^{2}} / \frac{\pi x 1.22^{2} \lambda^{2} q^{2}}{\delta^{2}}\right) 2 Q t \geq 25
$$

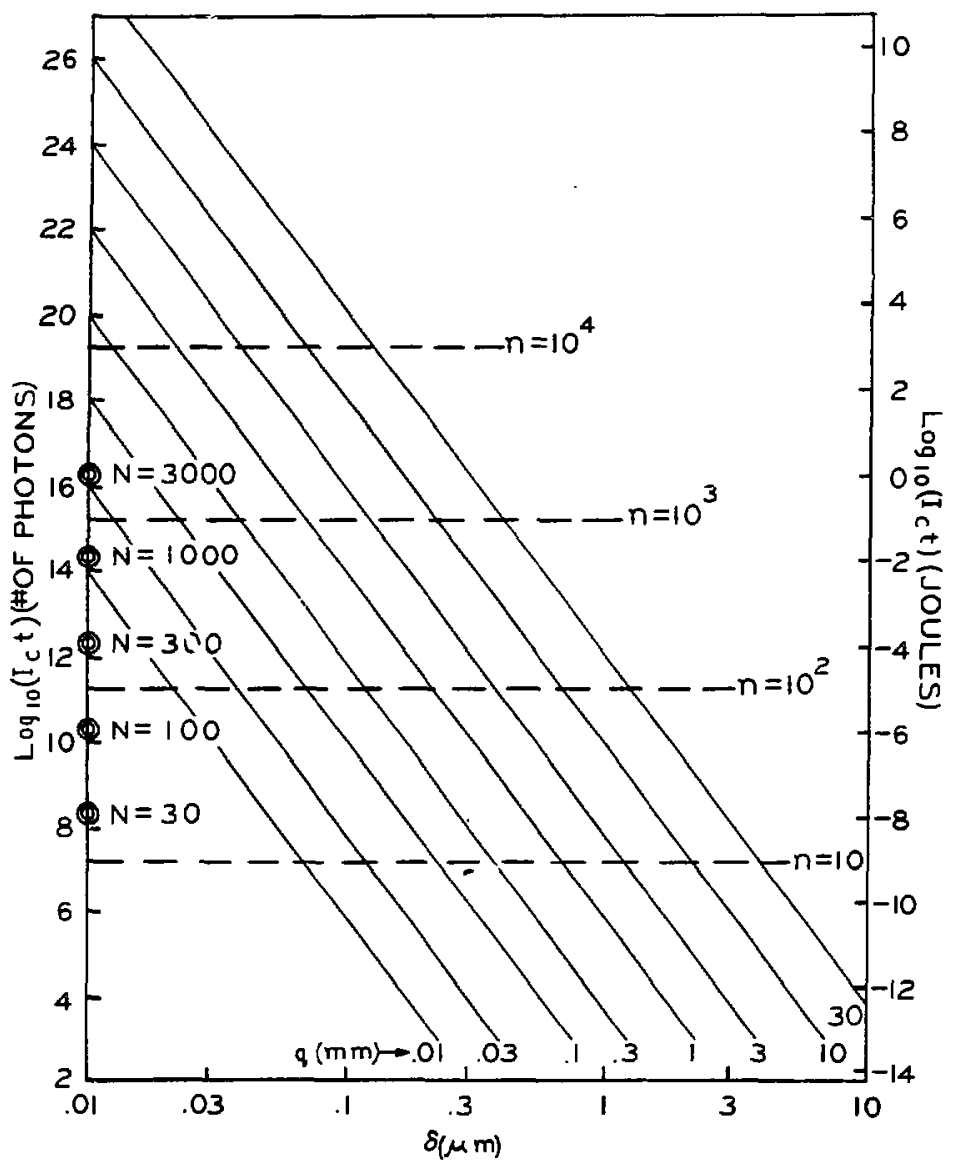

Fig. 7. Calculated fluence needs of Gabor hoiograms with various parameters. For all cases $\lambda=30 \AA$. $q$ is the sample-hologram distance, $I_{c} t$ is the fluence, $\delta$ is. the resolution element size in the sample, $n$ is the number of rings in the recorded zone plate pattern. The fluences needed for Fourier Transform holograms of $\delta$ are shown as double circles labelled with $N$ values where $\mathrm{N}^{2}$ is the total number of resolution elements in the sample 
If we now write $b$ as NS then we get

$$
I_{c} t \geq K_{3} N^{4}
$$

where $\mathrm{K}_{3}=2.9 \times 10^{2}$. We notice that the required fluence is independient of $\delta, q$ and even $\lambda$ and depends only on $N$ which is a measure of the field of view. Some fluence values from (24) are entered on Fig. 7. We notice that the Folrier Transform values are quite encouraging and are without the obligation to miniaturize which is implied in the zone plate cases. The lower information content of Fourier Transform holograms arises because $f$ irmation and reconstruction are with spherical waves and therefore it is $n$ it necessary to store 'lens' information in the hologram.

In order to give a feeling for the extent to which real sources can approach the flux needs expressed in Fig. 7 we present in Fig. 8 some estimates of the coherent power of various synchrotron radiation sources and some other sources which have been used for $x$-ray holography. One can easily see that the older sources were far short of achieving the beams needed for high resolution holography but that the newest ones offer great promise.

\section{Discussion}

We have considered many of the limitations involved in holographic imaging and we can now make some judgments on the best strategies for approaching our goal which is three dimersional imaging of biological material with a resolution of 100-200 \&. First we note that there are a number of reviews (21-24) of this general question in the literature and there is no concensus as to the preferred approach. There are different presumptions about available sources and different interests in regard to wavelength. Some authors regard good $x$-ray lenses as unavailable by definition. Others regard them as something to work toward.

We take the availability of both synchrotron radiation and undulator radiation as given. We takt: the 30 \& region as the one of prime interest for biology. We also assume that some zone plates are available with resolution around $0.1 \mu \mathrm{g}$ and that better ones will be available soon.

In light of these conditions we choose' to make the following first steps (i) to proceed immediately to make what holograms we can with techniques that are on hand, ( $i i)$ to move toward the high resolution regime via the Fourier Transform approach. (i) involves making Gabor holograms because these need the least technology. (ii) gives us the following advantages (a) it is possible to use photographic recording and reconstruct in the visible (b) one can move ahead readily using existing zone plates as beam forming optics while still looking for an improved reference source through development of special pinholes, etc. (c) future detector developments involving computer reconstruction can be carried out using devices that read directly to the computer in spite of the relatively low resolution of such devices (d) aberrations $(25,26)$ are much less in Fourier Transform holograms than in other types. 


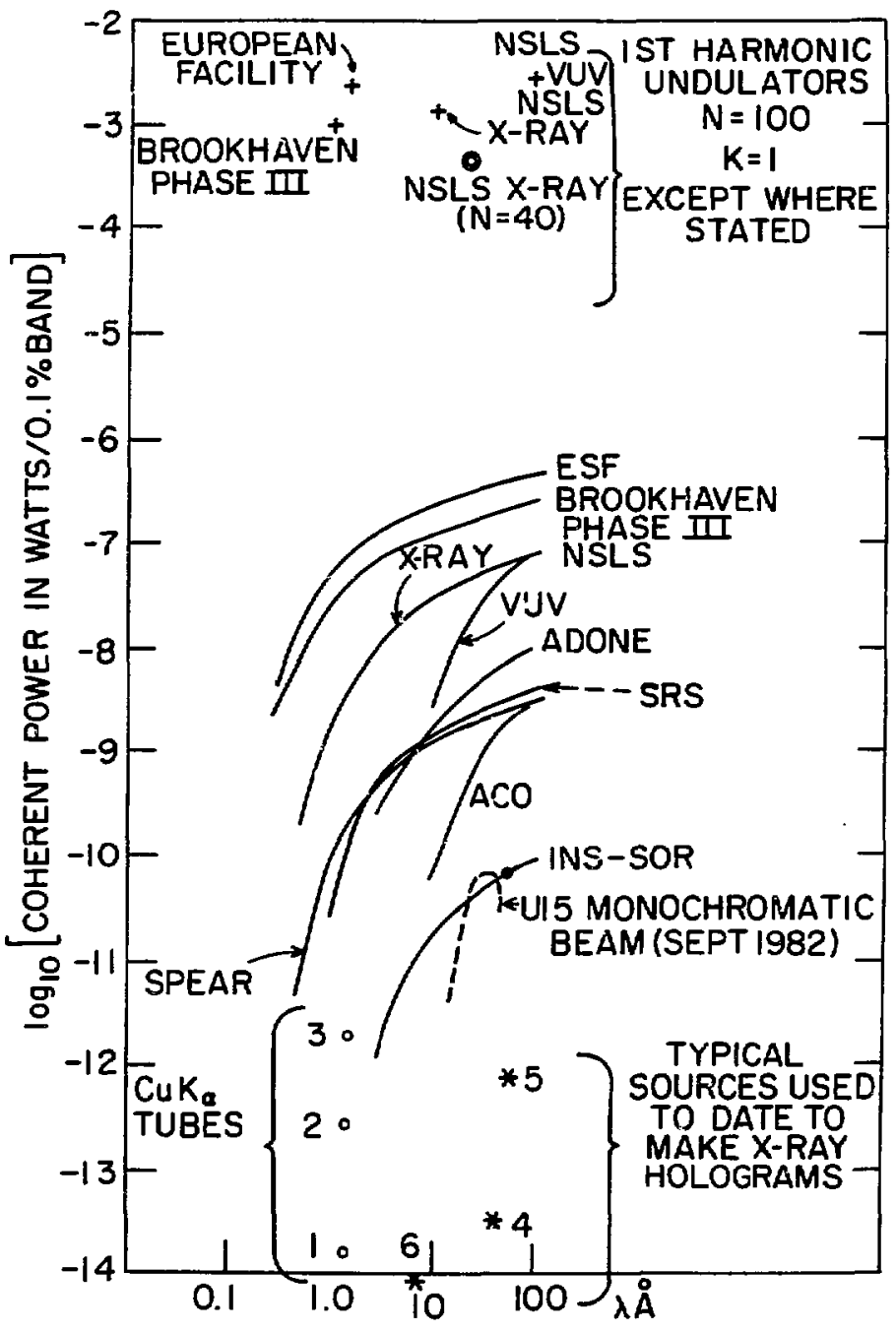

Fig. 8. Coherent power output of various sources. The numerical labels are to be interpreted as follows: (1) sealed CuR tube, $2 \mathrm{~kW}, 50 \mathrm{kV}$, (2) high power rotating anode CuR tube, $50 \mathrm{~kW}, 50 \mathrm{kV}$, (3) microfocus rotating anode Cuk tube, $3.5 \mathrm{~kW}, 50 \mathrm{kV},(4,5$ and 6) x-ray holography experiments due to Reuter and Mahr (29), Aoki and Kikuta (30) and Aoki et al (31), respectively. The double circle represents an undulator presently under construction at Brookhaven for use on the NSLS $x$-ray ring

We consider our plans for the future in detail in another publication (25). For the present we will report our experimental work to date and give some ideas on how we are beginning to understand it.

\section{Experimental Results}

We have recorded a number of Gabor Holograms using the arrangement shiwn in Figs. 9 and 10. Light from the National Synchrotron Light Source $750 \mathrm{MeV}$ 


\section{PHOTOGRAPHIC \\ PLATE \\ (KODAK I3I)}

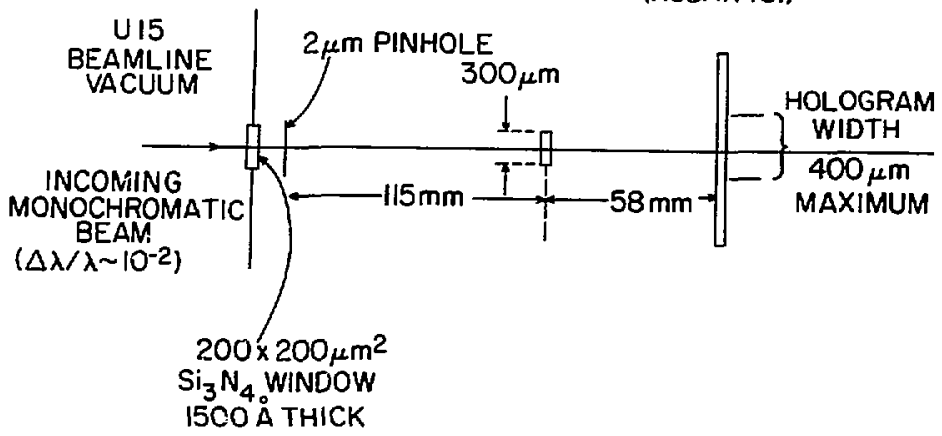

Fig. 9. Experimental geometry used to make Gabor holograms

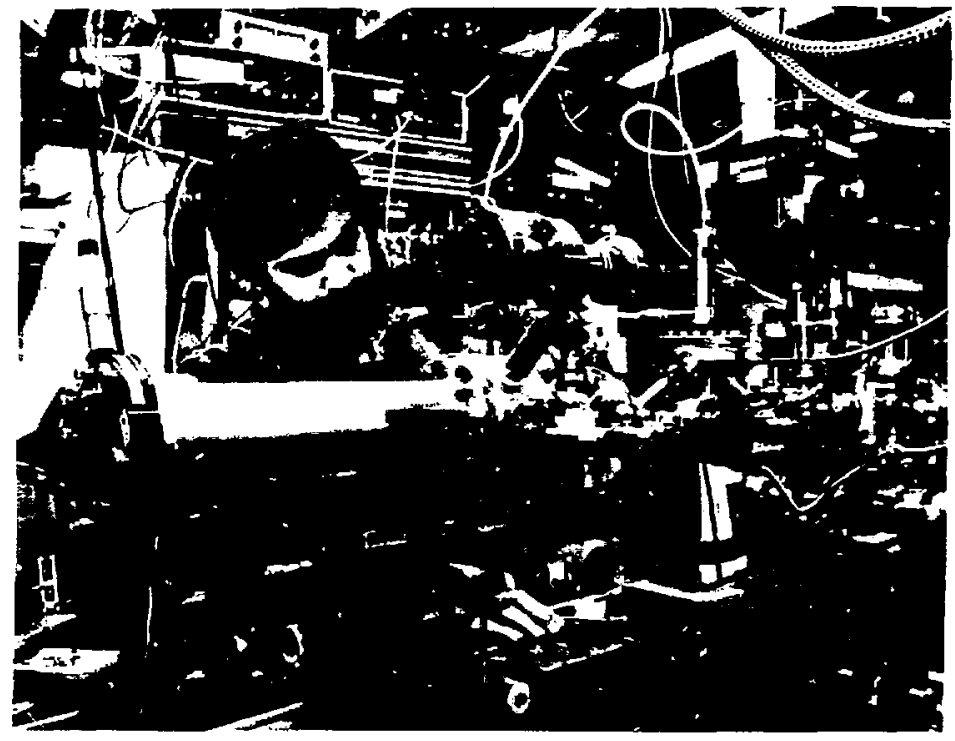

Fig. 10. U15 beamline with holography experiment in place

storage ring was roughly mongchromatized using the U15 beamline system (27). The light (of wavelength $31 \AA$ ) fully illuminated a $200 \times 200 \mu \mathrm{m}^{2} \mathrm{Si}_{3} \mathrm{~N}_{4}$ window which formed the end of the U15 vacuum system. In the holography chamber a $2 \mu$ pinhole was illuminated and used as a source of spatially 
coherent radiation. This illuminated the sample and also provided the reference beam by transmission through the sample. The holograms were recorded on Kodale type 131-02 high speed holographic film, which has a quoted resolution of $2500 \mathrm{l} / \mathrm{mm}$. Our samples were small objects of width $10 \mu \mathrm{m}$ or less so we see that in all cases recording was made in the far field of the sample. Baez (32) has given a prescription for calculating the resolution of the reconstruction of a Gabor hologram using a finite size source and a recording medium of finite resolution. Using this method for our arrangement predicts a resolution of about $0.8 \mu \mathrm{m}$.

Fig. 11 shows the hologram of several $12.5 \mu$ diameter wires. We see a rich fringe structure superimposed on the central peak of the Airy Disc of the 2 um pinhole. Fig. 12 shows a similar recording of a group of 3-5 $\mu \mathrm{m}$ diameter spherical, glass beads mounted on a $\mathrm{Si}_{3} \mathrm{~N}_{4}$ window. Again, a complex fringe structure is seen, this time, resembling more closely the 'blotchy' appearance of highly magnified visible light holograms. Fig. 13 shows the hologram of a single wire. It is very reminiscent of the analagous pictures taken in visible light by Thompson and Tvler (17) and of the famous firstever hologram taken in 1932 by Rellstrom $(28)$.

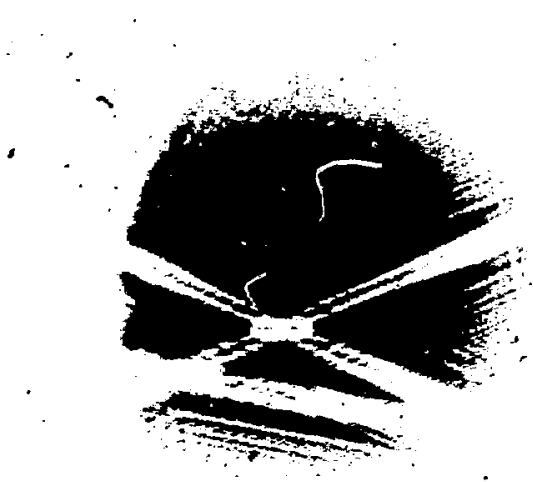

$\stackrel{100 \mu \mathrm{m}}{10}$

Fig. 11. Gabor hologram of cross wires $12.5 \mathrm{wn}$ diameter recorded on Rodak 131-02 film (quoted resolution $\sim 2500$ lines per $\mathrm{mm}$ ) using an exposure of 3 minutes at $100 \mathrm{~mA}$ beam current (about $50 \mu \mathrm{Joules} / \mathrm{cm}^{2}$ ). Magnification $\simeq 250 \mathrm{X}$ 


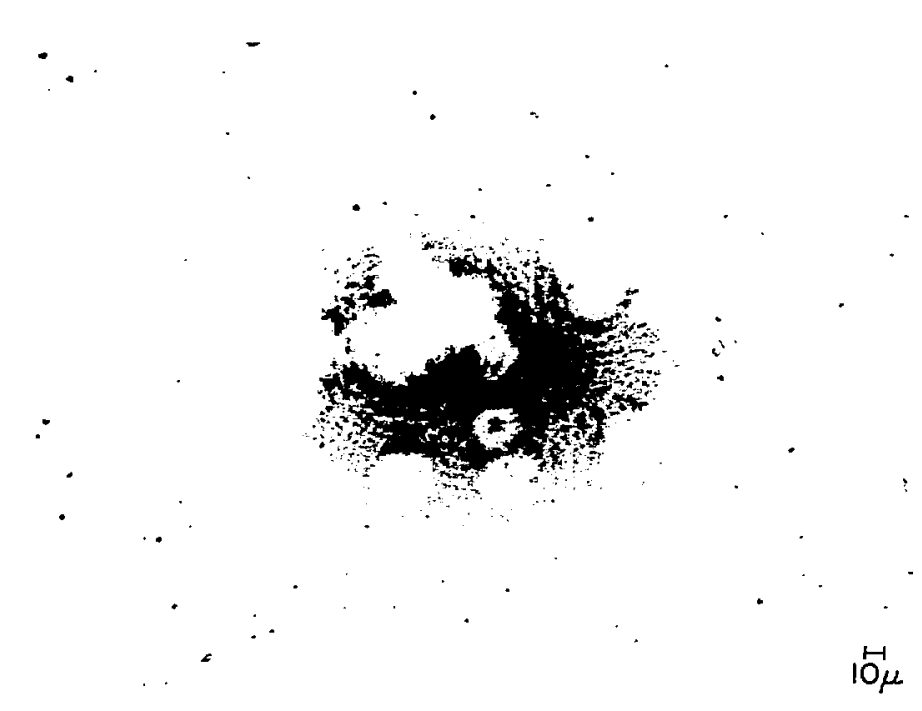

Fig. 12. Hologram of glass spheres 3-5 um diameter taken under similar conditions to Fig. 11

We have attempted to calculate the intensity distribution, $I(x)$, expected in a hologram such as Fig. 13. According to Thompson and Tyler this should be given by

$$
I(x)=1-\frac{4 a}{\sqrt{\lambda z}} \cos \left(\frac{\pi x^{2}}{\lambda z}-\frac{\pi}{4}\right) \operatorname{sinc}\left(\frac{2 \pi a x}{\lambda z}\right)+\frac{4 a^{2}}{\lambda z} \operatorname{sinc}^{2}\left(\frac{2 \pi a x}{\lambda z}\right)
$$

where $a$ is the radius of the wire, $z$ the sample to hologram distance and $x$ the co-ordinate in the detector perpendicular to the wire. This formula assumes uniform plane wave illumination which is not really appropriate for our case. To make some allowance for this we have folded a Gaussian of rough ly the right width into (24). The resulting cel lculated form is shown in Fig. 14 together with a microdensitometer trace of Fig. 13. We see that without any further attempts at a more realistic modal we obtain reasonable qualitative agreement. A more careful analysis of this question is present $1 y$ in progress.

In addition to attempting model calculations we are also trying to reconstruct our $x$-ray holograms using He:Cd laser light $(\lambda=4166 \AA)$. These eforts are just beginning but we have had some successes and one is 


\section{$\mathrm{I} 1 \mathrm{O} \mu$}

Fig. 13. Similar to Fig. 11 using just a single wire

illustrated in Fig. 15. We see a fairly good reconstruction of the crossed wires used to make the hologram in Fig. 11 .

\section{Conclusion}

We have reviewed the physics of zoft $x$-ray interactions with matter that could lead to imaging applications. We have given an outline of the operation of the holographic method especially emphasizing the geometries with promise for $x$-ray applications. We have considered the degree of spatial and temporal. coherence and the flux needed for successful imaging and have pointed out the promise of high brightness undulator sources in this connection. In the light of the general characteristics of the hologram forming methods we have concluded that for immediate use the Gabor geometry is hest and for the medium term the Fourier Transform offers the best chance of approaching the goal of high resolution biological imaging in three dimensions.

We have reported some results of our first attempts to make holograms with soft $x$-rays. These are of objects which, although uninteresting in themselves, allow rehearsal of the technique. Being simple they also allow comparison with theory and we have been qualitatively successful in achieving agreement with the calculated hologram intensity and with the expected geometry of the reconstructed image. 


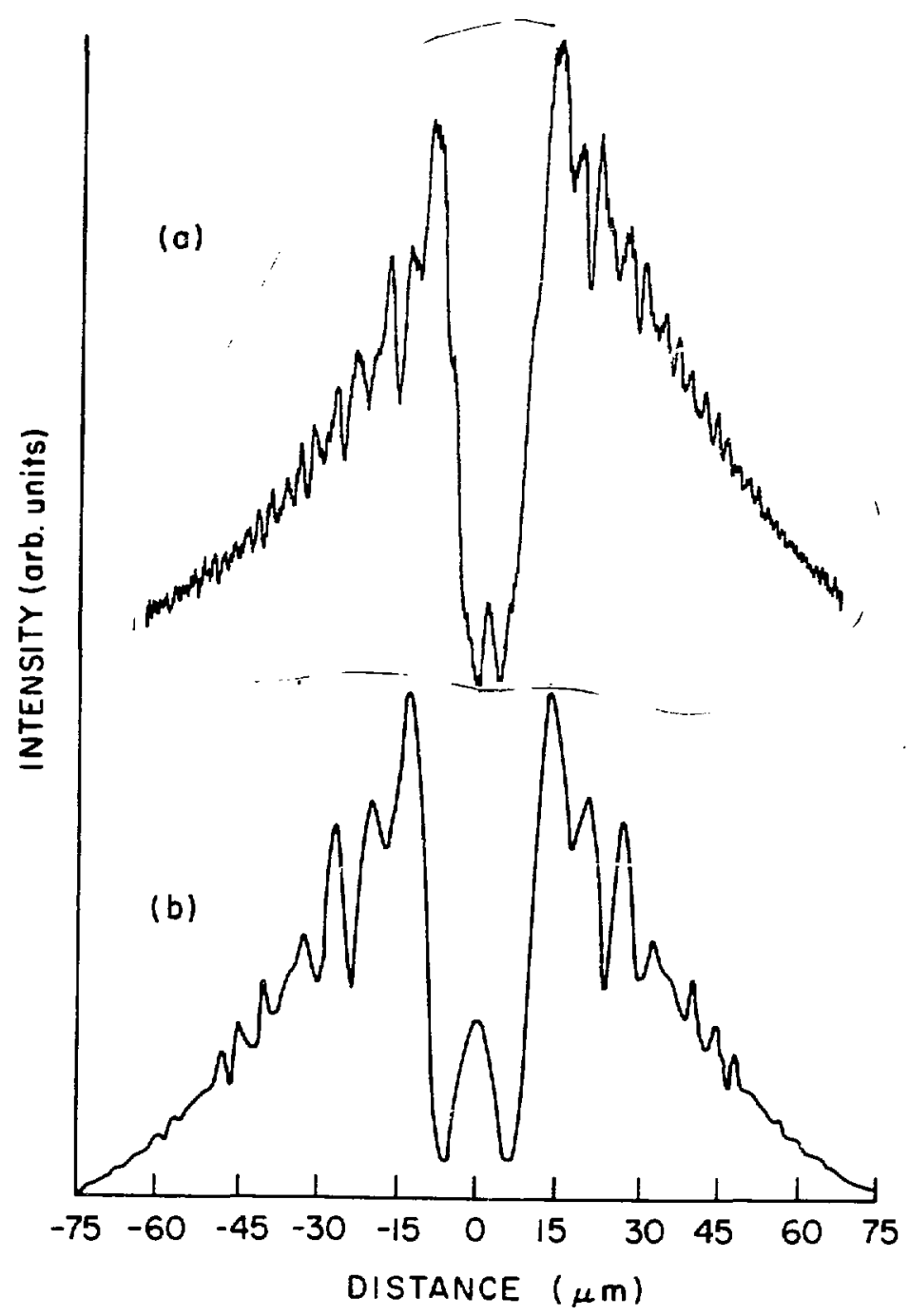

Fig. 14. (a) Microdensitometer trace of Fig. 13. (b) Computed plot of (24) folded with a Gaussian of approximately the right width to allow for the illumination profile of Fig. 13 


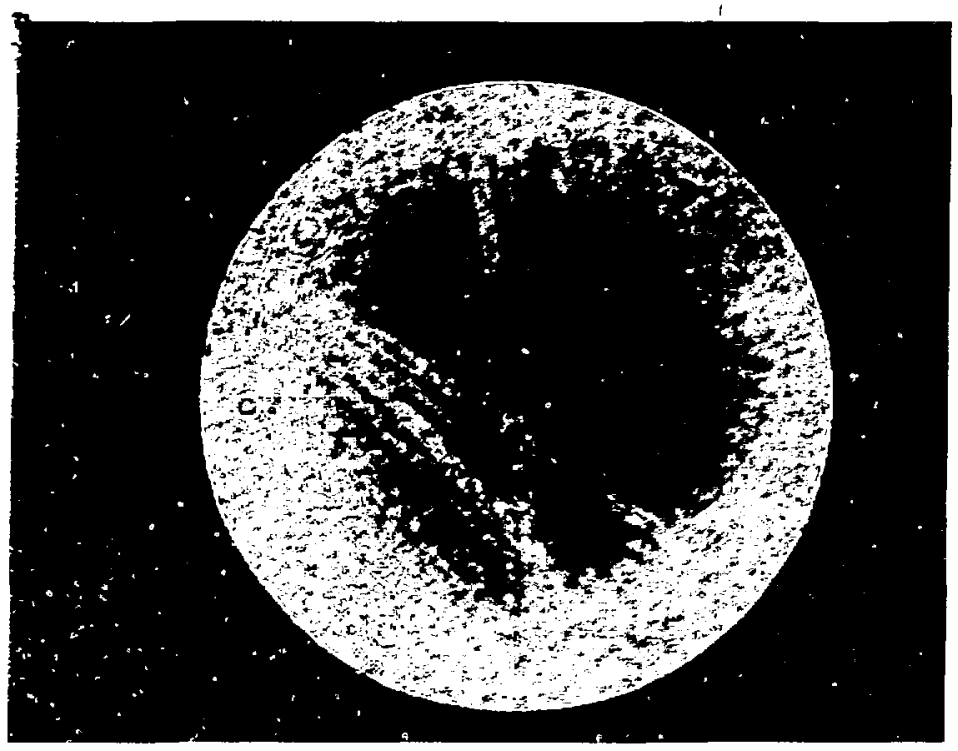

Fig. 15. Reconstruction of the hologram shown in Fig. 11 using He:Cd laser light $(\lambda=4166 \AA)$

\section{Ack now ledgments}

The author wishes to express his indebtedness to M. Iarocci of Brookhaven Naticnal Laboratory for creating the holographic experimental system, to $\mathrm{H}$. Rarback and J. Renney of the State University of New York at Stony Brook for providing the U15 beamline and to J. Kirz also of Stong Brook for continuing discussions, advice and intellectual stimulation.

\section{References}

1. J. Rirz and D. Sayre: Soft X-ray microscopy of biological specimens in Synchrotron Radiation Research, H. Winick and S. Doniach, Eds. Plenum, New York (1980)

2. G. Schmah 1 et a 1: X-ray microscopy in Uses of Synchrotron Radiation in Biology, H.B. Sturman, Academic, London, 1982 .

3. J. Renney et a1: Proceedings of the Brookhaven Conf. on Synchrotron Radiation Instrumentation. Sept. 1983. 
4. D. Sayre: Prospects for long wavelength $x$-ray microscopy and diffraction in Imaging Processes and Coherence in Physics, Fd. M. Schlenker et a1, Proceedings Les Houches 1979, Springer-Verlag, New Yorte (1979)

5. J.C. Solem ąnd G.C. Baldwin: Science, 218, 4569, 229 (1982)

6. See for example N.F. Mott and H. Massey: The Theory of Atomic Collisions, 2nd Edition, Oxford, 1950, p. 116

7. B.L. Henke: Monterey Bonf. on Low Energy X-Ray Diagnostics, Proc. AIP 75, $146(1981)$

8. B.L. Bragg: Nature, 143, 678 (1939)

9. See for example: Optica1 Transforms, H. Lipson, Academic, London (1972)

10. R.J. Collier, C.B. Burckhardt and L.H. Lin: Optical Holography, Academic, 1971

11. H.M. Smith: Principles of Holography, Wiley, New York, 1975

12. F.T.S. Yu: Optical Information Processing, Wiley, New York (1983)

13. J.W. Goodman: Introduction to Fourier Optics, McGraw Hi11, San Francisco (1968)

14. D. Gabor: Proc. Roy. Soc. A197, 454 (1949)

15. E.N. Leith, J. Upatneiks: J.0.S.A., 52, 1123 (1962)

16. G.W. Stroke: App 1.. Phys. Lett. $\underline{6}, 201$ (1965)

17. G.A. Tyler, B.J. Thompson: Optica Acta 23, 685 (1976)

18. C. Pellegrini: Brookhaven National Laboratory Report 非1684 (1982); also Optical Society of America, Topical Meeting on Free Electron Generation of Extreme Ultraviolet Coherent Radiation, Brookhaven, Sept. 19, 1983; and Department of Energy Field Task Proposal (1983) bv C. Pellegrini, et al

19. M. Born and E. Wolf: Principles of Optics, 3rd Ed., Pergamon, Oxford, p. 508 (1965)

20. M. R. Howe11s, J. Rirz, S. Krinsky: A beamline for experiments with coherent soft $x$-rays, Brookhaven Laboratory Report \#32519 (1982)

21. V.V. Aristov and G.A. Ivanova: J. App1. Cryst. 12, 19 (1979)

22. G.L. Rogers and J. Palmer: J. Microsc (Oxford), 89, 125 (1969)

23. J. Solem: High intensity $x$-ray holography: An approach to high resolution snapshot imaging of biological specimens. Los Alamos National Laboratory Report LA-9508-MS (1982)

24. A.M. Kondratenko, A.N. Skrinsky: Opt. Spectrose. (USSR) 42,189 (1977) 
25. SPIE Meeting on Advances in Soft $X$-Ray Science and Technology, Brookhaven, Oct. 17, 1983. To be published as Proc. SPIE, Vo1. 447

26. R.W. Meier, J.0.S.A. 56, 219 (1966)

27. J. Kirz et al, This volume

28. G. Kel1strom: Nov. Acta-Reg. Soc. Sci., Uppsaliensis $\underline{8}, 5$ (1932)

29. B. Reuter and H. Mahr: J. Phys. E, 9, 746 (1976)

30. S. Aoki, S. Kikuta: Jap. J. App 1. Phys. 11, 1857 (1972)

31. S. Aoki, et a1: Jap. J. App 1. Phys. 13, 9, 1385 (1974)

32. A. Baez: J.0.S.A. 42,756 (1952) 